\title{
IMPACTO PSICOSOCIAL DE LAS MALOCLUSIONES EN NIÑOS DE 8 A 12 AÑOS ATENDIDOS EN LA CLÍNICA ODONTOLÓGICA DE LA UNIVERSIDAD CATÓLICA DE CUENCA, 2019.
}

\author{
Psychosocial impact of malocclusions in children between 8 and 12 years of age \\ treated at the Dental Clinic of the Catholic University of Cuenca, 2019.
}

\author{
Romero-Espinosa José David ${ }^{* 1}$, Llanes-Serantes Maribel ${ }^{2}$, Salgado-Álvarez Rosa Emilia ${ }^{3}$. \\ ${ }^{1}$ Odontólogo Rural del Ministerio de Salud Pública del Ecuador. \\ 2 Docente de la Universidad Católica de Cuenca. Dra. Esp. en Ortodoncia. \\ 3 Odontóloga general de práctica privada. Azuay - El Oro, Ecuador. \\ *emi-s.a@hotmail.com
}

\begin{abstract}
Resumen
Objetivos: Determinar el impacto psicosocial de las maloclusiones en niños de 8 a 12 años atendidos en la Clínica Odontológica de la Universidad Católica de Cuenca. Materiales y métodos: La presente investigación es un estudio descriptivo, transversal, de campo, con una técnica observacional. La muestra consistió en 50 pacientes que fueron atendidos en la clínica odontológica de la Universidad Católica de ambos sexos con edades entre 8 a 12 años. Para la toma de datos se utilizó un cuestionario del impacto psicosocial de la estética dental (PIDAQ), contando con consentimiento y asentimiento informado. Resultados: Se evaluó el impacto psicosocial según el género obteniendo una significancia de p=0.0001248. Conclusiones: Se determinó que en niños de 8 a 12 años las maloclusiones si tienen un impacto psicosocial y que es significativamente diferente según el sexo, afectando mayormente al género femenino.
\end{abstract}

Palabras clave: Psicosocial, maloclusiones, PIDAQ.

\begin{abstract}
Aim: Determine the psychosocial impact of malocclusions in children between 8 and 12 years old treated at the Dental Clinic of the Catholic University of Cuenca. Material and methods:The present research is a descriptive, cross-sectional, field, observational study. The sample consisted of 50 patients who were treated at the dental clinic of the Catholic University, of both sexes, aged between 8 and 12 years. For data collection a questionnaire on the psychosocial impact of dental aesthetics (PIDAQ) was used, with informed consent and assent. Results: The psychosocial impact was evaluated according to gender, obtaining a significance of $p=0.0001248$. Conclusions: It was determined that in children between 8 and 12 years old, malocclusions do have a psychosocial impact and that it is significantly different according to sex, affecting more the female gender.
\end{abstract}

Key words: Psychosocial, malocclusions, PIDAQ.

\section{Introducción}

Las maloclusiones son alteraciones funcionales, estéticas, anatómicas, de origen genético, dentario o traumático, que involucran a los tejidos blandos y duros del complejo estomatognático. ${ }^{1-3}$

La infancia y primera adolescencia es un período decisivo para el desarrollo mental, emocional y social de un individuo, estos años son fundamentales para una vida plena y saludable, sin embargo, este desarrollo puede ser afectado por diferentes factores externos e internos como son las enfermedades orales, entre ellas, las maloclusiones, teniendo un efecto negativo en los ámbitos emocionales, sociales, afectando su autoestima, peso, crecimiento, aprendizaje, comunicación, calidad de vida. ${ }^{4,5}$ 
El primer paso para entender un problema de salud es plantear una pregunta de investigación acerca de la prevalencia del problema. ${ }^{6}$ A pesar de que las maloclusiones presentan una alta prevalencia incidencia y son considerados un problema de salud pública, ${ }^{7-15}$ no son muchos los estudios que se enfocan desde la perspectiva psicosocial y su impacto en estos individuos, ${ }^{16-20}$ fue por esta razón que se planteó el objetivo del presente estudio, determinar el impacto psicosocial de las maloclusiones en niños de 8 a 12 años atendidos en la Clínica Odontológica de la Universidad Católica de Cuenca, en el año 2019.

\section{Materiales y métodos}

La presente investigación es un estudio descriptivo, transversal, de campo, con una técnica observacional. ${ }^{6}$ El tamaño de la población fue de 50 pacientes de ambos géneros de 8 a 12 años que fueron atendidos en la clínica odontológica de la Universidad Católica de Cuenca, siendo la muestra del total de la población.

El presente estudio no implicó conflictos bioéticos, debido a que se ejecutó sobre datos previamente obtenidos, en los cuales los padres o tutores legales fueron informados de manera escrita de los objetivos y metodología del estudio, recibiendo un consentimiento aprobado por los mismos y un asentimiento de los niños. Se indicó un compromiso de confidencialidad de los datos obtenidos.

La toma de datos se realizó mediante el cuestionario de impacto psicosocial PIDAQ. ${ }^{19}$ Esta evaluación estuvo dividida en cuatro subdominios: (Tabla 1)

El puntaje mayor del PIDAQ son 92 puntos, de 0 a 46 se define como bajo impacto, mientras que de 47 a 92 se define como alto impacto. El subdominio de Autoconfianza Dental (AC) está manejado de manera positiva, mientras su puntuación sea más alta menos impactó representa. Los subdominios Impacto Social (IS), Impacto Psicológico (IPS) y Preocupación por la Propia Estética (PPE) están manejadas de manera contraria, por lo tanto, si la puntuación es más alta el impacto es mayor y tendrá una afectación negativa.

Para la valoración de cada pregunta se aplicó una escala de Likert de 5 puntos que van de 0 a 4 :

$$
\begin{aligned}
0 & =\text { Nada } \\
1 & =\text { Un poco. } \\
2 & =\text { Algo. } \\
3 & =\text { Mucho. } \\
4 & =\text { Muchísimo. }
\end{aligned}
$$

La puntuación total se obtuvo sumando las puntuaciones de los todos los ítems y se procedió a hacer el análisis de los resultados.

\section{Resultados}

En el presente estudio, el impacto psicológico de las maloclusiones fue evaluado en 50 niños de 8 a 12 años atendidos en la Clínica Odontológica de la Universidad Católica de Cuenca. Al analizar la distribución de la muestra se observó que, el $42 \%(\mathrm{n}=21)$ pertenecían al sexo femenino, y el $58 \%(n=29)$ al sexo masculino.

Al analizar el puntaje total de las respuestas del cuestionario (PIDAQ), se determinó que, en cuanto al impacto psicosocial de las maloclusiones, en este estudio si hubo relación entre la maloclusión y el impacto psicosocial obteniendo un total de 51/92 (Tabla 2). Al analizar por separado cada subdominio, el de autoconfianza estética dental (CA) por ser planteado de manera positiva, su puntuación de 7/24 demuestra que si tuvo impacto, los subdominios: impacto social 20/32, impacto psicológico $17 / 24$ y preocupación por la propia estética dental 7/12, al ser inversamente proporcionales al anterior, mientras mayor puntaje mayor impacto negativo, demostrando que las maloclusiones si tienen un impacto psicosocial negativo en los diferentes ámbitos de la vida de los pacientes (Tabla 2).

En la distribución porcentual de respuestas del PIDAQ en la escala de Likert se encontró que la respuesta con menos contestación fue "nada" $9 \%$, seguida de "muchísimo" $11 \%$, continuando con "poco" $18 \%$, "mucho" $26 \%$, finalizando con "algo" $36 \%$ (Tabla 3).

La distribución de la frecuencia de respuestas del (PIDAQ) en la escala de Likert según el sexo demostró que los valores no son equitativos, por lo cual se evidencia que el impacto psicosocial de las maloclusiones afecta de manera diferente a ambos sexos, teniendo mayor impacto negativo en el sexo femenino.

\section{Discusión}

La medición de la calidad de vida relacionada a la salud bucal tiene una relevancia actual y se ha ido investigando en los últimos 20 años a nivel mundial ${ }^{20-29}$ y en especial en Sudamérica en la última década. ${ }^{30-37}$ En esta investigación sobre impacto psicosocial de las maloclusiones en niños de 8 a 12 años atendidos en la clínica odontológica de la Universidad Católica de Cuenca en la distribución de la muestra, presentó que un $58 \%$ de la misma pertenecían al sexo masculino, al igual que en los estudios realizados en Chile por Solís, Perú por Miranda, Ecuador por Saltos, los cuales coincidieron en un predominio del sexo masculino. Esta semejanza se manifestó a pesar de que las muestras seleccionadas en todos estos estudios fueron mayores a las analizadas en esta investigación. ${ }^{26,36,37}$

En relación con la distribución porcentual del subdominio de autoconfianza dental (CA), se obtuvo 


\begin{tabular}{lcc}
\hline \multicolumn{1}{c}{ SUBDOMINIO } & NÚMERO DE ITEMS & PUNTOS \\
\hline Autoconfianza dental (AC) & 6 & 24 \\
Impacto social (IS) & 8 & 32 \\
Impacto psicológico (IPS) & 6 & 24 \\
Preocupación por la Propia Estética & 3 & 12 \\
TOTAL & 23 & 92 \\
\hline
\end{tabular}

Tabla 1: Subdominios del PIDAQ y puntaje.

\begin{tabular}{lccccc}
\hline \multicolumn{1}{c}{ SUBDOMINIOS } & \multicolumn{2}{c}{ PUNTAJE PIDAQ } & \multicolumn{2}{c}{$\begin{array}{c}\text { PUNTAJE } \\
\text { REFERENCIAL }\end{array}$} \\
\hline & $\begin{array}{c}n \\
\text { preguntas } \\
\text { Autoconfianza dental (AC) }\end{array}$ & puntaje & $\%$ & $\begin{array}{c}\text { valor } \\
\text { mínimo }\end{array}$ & $\begin{array}{c}\text { valor } \\
\text { máximo }\end{array}$ \\
Impacto social (IS) & $\mathrm{n}=300$ & 7 & $29 \%$ & 0 & 24 \\
Impacto psicológico (IPS) & $\mathrm{n}=300$ & 20 & $63 \%$ & 0 & 32 \\
Preocupación por la Propia Estética & $\mathrm{n}=150$ & 7 & $71 \%$ & 0 & 24 \\
TOTAL & $\mathrm{n}=1150$ & 51 & $55 \%$ & 0 & 12 \\
\hline
\end{tabular}

Tabla 2: Resultado del puntaje PIDAQ por subdominios.

\begin{tabular}{lcccccc}
\hline \multicolumn{1}{c}{ SUBDOMINIOS } & \multicolumn{7}{c}{ ESCALA DE LIKERT } \\
\hline & nada & poco & algo & mucho & muchísimo & TOTAL \\
Autoconfianza dental (AC) & $27 \%$ & $49 \%$ & $22 \%$ & $2 \%$ & $0 \%$ & $100 \%$ \\
Impacto social (IS) & $1 \%$ & $10 \%$ & $44 \%$ & $35 \%$ & $10 \%$ & $100 \%$ \\
Impacto psicológico (IPS) & $0 \%$ & $6 \%$ & $40 \%$ & $30 \%$ & $24 \%$ & $100 \%$ \\
Preocupación por la Propia Estética & $9 \%$ & $8 \%$ & $38 \%$ & $35 \%$ & $10 \%$ & $100 \%$ \\
TOTAL PIDAQ & $9 \%$ & $18 \%$ & $36 \%$ & $26 \%$ & $11 \%$ & $100 \%$ \\
\hline
\end{tabular}

Tabla 3: Distribución porcentual de respuestas del PIDAQ por subdominios en la escala de likert.

que la respuesta más frecuente fue "un poco" con un $49 \%$ de la muestra, a diferencia de los estudios realizados por Solís y Miranda en los que la más frecuente fue "algo" con un $40 \%$ y $39 \%$, pero siendo coincidente en la respuesta menos 


\begin{tabular}{|c|c|c|c|c|c|c|c|c|c|c|c|c|}
\hline \multirow[t]{3}{*}{ SEXO } & \multicolumn{12}{|c|}{ ESCALA DE LIKERT } \\
\hline & \multicolumn{2}{|c|}{ nada } & \multicolumn{2}{|c|}{ poco } & \multicolumn{2}{|c|}{ algo } & \multicolumn{2}{|c|}{ mucho } & \multicolumn{2}{|c|}{ muchísimo } & \multicolumn{2}{|c|}{ TOTAL } \\
\hline & $\mathrm{n}$ & $\%$ & $\mathrm{n}$ & $\%$ & $\mathrm{n}$ & $\%$ & $\mathrm{n}$ & $\%$ & $n$ & $\%$ & $\mathrm{n}$ & $\%$ \\
\hline Masculino & 52 & $5 \%$ & 83 & $7 \%$ & 144 & $12 \%$ & 134 & $12 \%$ & 70 & $6 \%$ & 483 & $42 \%$ \\
\hline Femenino & 45 & $4 \%$ & 135 & $11 \%$ & 275 & $24 \%$ & 155 & $14 \%$ & 57 & $5 \%$ & 667 & $58 \%$ \\
\hline TOTAL PIDAQ & 97 & $9 \%$ & 218 & $18 \%$ & 419 & $36 \%$ & 289 & $26 \%$ & 127 & $11 \%$ & 1150 & $100 \%$ \\
\hline
\end{tabular}

Tabla 4: Frecuencias de respuestas del PIDAQ en la escala de Likert, según el sexo.

frecuente "muchísimo" con 5\% y $0 \%$ a igual que en esta investigación $0 \% .{ }^{26,36}$

En cuanto a la distribución porcentual del subdominio de impacto social (IS), la respuesta con mayor frecuencia "algo" con $44 \%$ y la de menor frecuencia "nada" con $1 \%$ mostrando una amplia diferencia con otros investigadores ya que en sus estudios la respuesta más encontrada fue "nada" con un $50 \%$ y $56.5 \%$ y menor encontrada fue "muchísimo" con $1 \%$ y $3.1 \% .^{26,36,37}$

Analizando la distribución porcentual del subdominio de impacto psicológico (IPS), se ha observado que la respuesta de menor frecuencia fue "nada" con un $0 \%$, contrario al estudio realizado en Chile por Solís, con menor frecuencia en la respuesta "muchísimo" con un $1 \%$ y la del estudio de Lima realizado por Miranda que fue "mucho" con el $7.55 \%$, en la respuesta más prevalente tampoco encontró similitud en ninguno de los estudios realizados. ${ }^{36,37}$

En cuanto a la distribución porcentual del subdominio de preocupación por la propia estética dental (CED), se arrojaron resultados semejantes a las respuestas del impacto psicológico, ya que no hubo similitudes con ningún estudio revisado; la respuesta con mayor frecuencia encontrada fue "algo", mientras que la respuesta de los estudios de Solís y Miranda fue "nada" con $45 \%$ y 56,85\% respectivamente; la respuesta con menor frecuencia en el estudio de Solís fue "muchísimo" con un $5 \%$ y el de Miranda fue "mucho" con $4.52 \%$. En este estudio a diferencia del estudio realizado por Miranda en el cual la respuesta con menor frecuencia fue "nada" con $9 \% .^{26,36,37}$

Al comparar la influencia del sexo en la frecuencia de respuestas del cuestionario (PIDAQ), se encontró que, si existe una diferencia significativa entre lo contestado por los hombres que, por las mujeres, $(\mathrm{p}=0,0001248)$ resultado que fue similar a lo encontrado por Saltos y por Solís y diferente a lo encontrado por Miranda en el cual el único aspecto que se observó influenciado por el sexo fue el impacto psicológico. $26,36,37$

\section{Conclusiones}

Al realiza reste estudio se determinó que en niños de 8 a 12 años las maloclusiones si tienen un impacto psicosocial negativo y que es significativamente diferente según el sexo, afectando mayormente al género femenino.

Conflictos de intereses: Los autores declaran no tener conflictos de intereses.

\section{Referencias}

1 Aliaga del Castillo A, Mattos Vela M. Maloclusiones en niños y adolescentes de caseríos y comunidades nativas de la amazonia de Ucayali, Perú. Rev. Perú. Med. Exp. Salud publica [Internet] v.28 n.1

2 Díaz Méndez H, Ochoa Fernández B, Paz Quiñonez L, Casanova K, Coca García Y. Maloclusiones en niños de la escuela Carlos Cuquejo del municipio Puerto Padre, Las Tunas. Medisur. [Internet] v.13 n.4

3 López Montes R, García Rocha A, Martín López A. Maloclusiones dentarias en alumnos de 9-12 años de la escuela primaria federal Ignacio Ramírez de Tihuatlan Veracruz. Universidad Veracruciana. [Internet] pag.87

4 Medina C. Prevalencia de maloclusiones dentales en un grupo de pacientes pediátricos. Acta odontol. Venez [Internet] v.48 n.1.

5 Ferreira V, Martins F. Oclusión y equilibrio de los dientes, clasificación de las maloclusiónes. en: Ferreira V, Hecht M. Ortodoncia diagnóstico y planificación clínica. Vol. 1. Sao Paulo. Artes Médicas Latinoamérica; 2002. p. 74-1

6 Villavicencio Caparó E, Cuenca León K, Vélez León E, Sayago Heredia J, Cabrera Duffau A. Pasos para la planificación de una investigación clínica. Oactiva ucacue. [Internet] v.1 n.1 
7 Ganan A. Estudio de la prevalencia de apiñamiento dentario en niños de 6 a 12 años de edad del Instituto Tecnológico 22 Superior Consejo Provincial de Pichincha período 2015. Universidad Central del Ecuador [Tesis]

8 Hernández J, Padilla M. Tratamiento temprano de la mordida cruzada anterior. Revisión de la literatura. Rev. Estomat. 2011; 19(2):40-47.

9 Beraud Osorio D, Sánchez Rodríguez M, Murrieta Pruneda J, Mendoza Nuñez V. Prevalencia y factores de riesgo de mordida cruzada posterior en niños de 4-9 años de edad en 24 ciudad Nezahualcóyotl. Bol. Med. Hosp. Infant. Mex. 2004; 61(2).

10 Pereira Rosa A, Viera de Souza B, Alves Herdy J. Mordida Cruzada Posterior. Revista Red de Ciudadanos en Saüde. 2017; 11(2).

11 Gregoret J, Tuber E. Ortodoncia y cirugía ortognática diagnóstico y planificación. en: Examen Bucodental. Barcelona 25 1997. p. 1(2); 49-61-460

12 Bordignon N. El desarrollo psicosocial de Erik Erikson. El diagrama epigenético del adulto. Rev. Lasallista. 2005; 2(2).

13 Marques 1, Ramos M, Paiva S, Pordeus I. Malocclusion: esthetic impact and quality of life among Brazilian 26 schoolchildren. Am J Orthod Dentofacial Orthop. 2006; 129(3):424.

14 Klages U, Bruckner A, Guld Y, Zentner A. Dental esthetics, orthodontic treatment, and oral health attitudes in young adults. Am J Orthod Dentofacial Orthop. 2006; 128 (4): 442

15 Samsonyanová L, Broukal Z. A systematic review of individual motivational factors in orthodontic treatment:facial attractiveness as the main motivational factor in orthodontic treatment. International Journal of Dentistry. 2014; 2014: 14

16 Newton J, Prabhu N, Robinson P. El impacto del aspecto dental en la apreciación de las características personales. Int J Prosthodont. 2003; 16: 429.

17 Henson S, Lindauer S, Gardner W, Shroff B, Tufekci E, Best A. Influence of dental esthetics on social perceptions of adolescents judged by peers. AJO-DO. 2011; 140 (3), 389395.

18 Cesarino M, Almeida T, Melo de Brito E, Castro C, Flavia 3 A, Martins S. Impact of oral health conditions on the quality of life of preschool children and their families: a crosssectional study. Health and Quality of Life Outcomes. 2014;12:55 Disponible en: http://www.hqlo.com/content/12/1/55.

19 Abanto J, Bönecker M, Raggio D. Impacto de los problemas 31 bucales sobre la calidad de vida de niños. Rev Estomatol Herediana. 2010; 20 (1): 38- 43.

20 Calderón G. Influencia de seis maloclusiones en la percepción de inteligencia, atractivo físico y habilidades interper- 32 sonales. [Tesis de grado]. Quito: Universidad San Francisco de Quito; 2013

21 Taylor K, Kiyak A, Huang G, Greenlee G, Jolley C, King G. Effects of malocclusion and its treatment on the quality of 33 life of adolescents. Am J Orthod Dentofacial Orthop. 2009;
136 (3): 382-392.

O’Brien K, Wright JL, Conboy F, Macfarlane T, Mandall N. The child perception questionnaire is valid for malocclusion in the United Kingdom. Am J Orthod Dentofacial Orthop. 2006; 129(4):536-540.

23 Otuyemi OD, Ogunyinka A, Dosumu O, Cons NC, Jenny J. Malocclusion and orthodontic treatment need of secondary school students in Nigeria according to the dental aesthetic index (DAI). Int Dent J 1999; 49: 203-210.

4 Aguirre V. Alteraciones de la autoestima provocadas por diferentes factores que influyen en la estética dentofacial y su incidencia en los estudiantes del primero, segundo y tercero de bachillerato de los colegios "Manuel Cabrera Lozano" y "La Salle", en el periodo Abril-Septiembre 2011". Tesis previo a optar por el título de odontólogo. Universidad Nacional de Loja. Loja (Ecuador). 2011: 52-69

25 Inda D. Evaluación de la autopercepción de la estética dental e impacto psicosocial en pacientes sometidos a blanqueamiento dental, seguimiento de 9 meses. Tesis previa a optar por el título de Cirujano-Dentista. Universidad de Chile. 2016.

26 Saltos M. Maloclusiones y su influencia psicosocial en las / los estudiantes de 9 a 15 años de edad, de la unidad educativa " Liceo Policial“" de la ciudad de Quito, en el periodo 2015-2016. [tesis]. Universidad Central del Ecuador; 2016. $72 \mathrm{p}$.

27 Gutiérrez Y, Expósito I, Jiménez I, Estrada V, Martín L. Presencia de maloclusiones en adolescentes y su relación con determinados factores psicosociales. Archivo Médico de Camagüey 2008 [internet][citado 13 octubre 2016: 12(5):1-10.

28 Mafla A. Barrera D, Muñoz G. Maloclusión y necesidad de tratamiento ortodóntico en adolescentes de Pasto, Colombia. Rev Fac Odontol Univ Antioq. 2011; 22(2): 173-85

29 Puertes N. Necesidad de tratamiento ortodóncico e impacto psicosocial de la estética dental en los adolescentes de la comunidad valenciana. Tesis doctoral. Departamento de Fisiopatología del Aparato Estomatognático. Universidad de Valencia; 2013.

0 Bourzgui F, Serhier Z, Sebbar M., Diouny S, Bennani M, Ngom P. Adaptation and validation of the Moroccan Arabic versión of the Psychosocial Impact of Dental Aesthetics Questionnaire (PIDAQ). The Saudi dental Journal. 2015, 27:180 - 186.

1 Montiel JM, Bellot C, Almerich JM. Validation of the psychosocial impact of dental aesthetics questionnaire (Pidaq) in Spanish adolescents. Med Oral Patol Or Bucal 2013 Jan 1; 18(1) 168-73.

32 Paula DF, Santos NC, da Silva ET, Nunes MF, Leles C. . Psychosocial impact of dental esthetics on quality of life in adolescents.. The Angle orthodontist. 2009,79(6):1188 1193.

Twigge E, Roberts R, Jamieson L, Dreyer C, Sampson W. The psycho-social impact of malocclusions and treatment 
expectations of adolescent orthodontic patients. European Journal of Orthodontics. 2016, 38(6):593 -601

34 Yi S., Zhang C.,Ni C.,Quian Y.,Zhang ., Psychosocial impact of dental aesthetics and desire for orthodontic treatment among chinese undergraduate students. Dove Press Journal.2016,10: 1037 - 1042 .

35 Cartes Velásquez, Ricardo \& Araya, Eric \& Valdés, Carolina. (2010). Maloclusiones y su Impacto Psicosocial en Estudiantes de un Liceo Intercultural. International Journal of Odontostomatology. 4. 65-70.

36 Solís B; Catril R; Parra F; Quemener J. Impacto psicosocial asociado a presencia de maloclusiones en estudiantes de primero a cuarto medio de los colegios marina de chile y santa luisa, concepción 2014. . [tesis]. Universidad Concepción; 2014. 35 p.

37 Miranda A. Impacto psicosocial de la maloclusión en adolescentes de una institución educativa de Lince - Lima. [tesis]. Universidad Nacional Mayor de San Marcos; 2017. $107 \mathrm{p}$.

Recibido: 23 de septiembre de 2020

Aceptado: 01 de diciembre de 2020 